\title{
The evaluation of cesarean section rates in accordance with Robson Ten-Group Classification System and the data of perinatology (tertiary center)
}

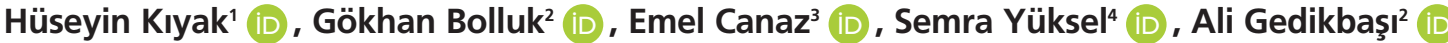 \\ 'Gynecology and Obstetrics Clinic, Istanbul Kanuni Sultan Sïleyman Training and Research Hospital, Istanbul, Turkey \\ ${ }^{2}$ Perinatology Department, Gynecology and Obstetrics Clinic, Istanbul Kanuni Sultan Siileyman Training and Research Hospital, Istanbul, Turkey \\ ${ }^{3}$ Department of Obstetrics and Gynecology, Faculty of Medicine, İstanbul Bilim University, İstanbul, Turkey \\ ${ }^{4}$ Gynecology and Obstetrics Clinic, Istanbul Taksim GOP Training and Research Hospital, İstanbul, Turkey
}

\begin{abstract}
Objective: To evaluate separately the impacts of clinical data of pregnant women and perinatology on Robson Ten-Group Classification System in our hospital.

Methods: After the data of pregnant women and perinatology outcomes between 2015 and 2019 were obtained digitally from the system and distinguished from each other, all groups were evaluated by comparing them in accordance with the suggestions of the WHO.

Results: A total of 24,240 (42.2\%) out of 57,402 labors were carried out by cesarean section. Of 42,500 ( $74 \%$ of all labors) pregnant women composing the pregnant women group, 15,025 (35.4\%) delivered by cesarean section. Of 14,902 (26\% of all labors) pregnant women who were followed up in the perinatology clinic, 9215 $(61.8 \%)$ delivered by cesarean section. The hospital receives a high rate of multiparous case application (39.2\%). The cesarean section is performed in $99.8 \%$ of the patients with previous history of cesarean section. The rate of pregnant women who admitted for breech presentation was lower than expected, and the rate of cesarean section was $96.8 \%$ for nulliparous breech presentations and $88.6 \%$ for multiparous breech presentations. The rate of pregnant women who admitted for multiple pregnancy was $6.1 \%$, and the rate of cesarean section for these cases was $90.1 \%$. The rate of pregnant women who were followed up in the perinatology clinic, admitted for various reasons, particularly for preeclampsia, and had preterm labor was $25.1 \%$, and the rate of cesarean section for these cases was $66.1 \%$.

Conclusion: The cesarean rates associated with perinatology clinic/tertiary center are much higher. In order to keep the rates of primary cesarean section within reasonable levels, the practices of persistent labor induction should be avoided, cesarean section should not be recommended for tubal ligation procedure, and external cephalic version should be tried in breech presentation cases in appropriate patients.
\end{abstract}

Keywords: Robson Ten-Group Classification System, perinatology/tertiary clinic.

\section{Özet: Sezaryen oranlarını Robson On Gruplu Sınıflandırma Sistemi ve perinatoloji (tersiyer merkez) verileri doğrultusunda konuşmak}

Amaç: Hastanemizde gebe ve perinatoloji klinik verilerinin Robson On Gruplu Sinıflandırma Sistemi (ROGSS) üzerine etkilerini ayrı ayrı değerlendirmek.

Yöntem: 2015-2019 verileri dijital olarak çıkarılıp sistem üzerinden gebe ve perinatoloji sonuçları ayırt edildikten sonra, bütün gruplar Dünya Sağlık Örgütü önerileri doğrultusunda karşılaştırılarak incelendi.

Bulgular: Toplam 57.402 doğumun 24.240 tanesi (\%42.2) sezaryen ile doğurtulmuştur. Gebe grubunu oluşturan 42.500 gebenin (tüm doğumların \%74'ü) 15.025 tanesi sezaryen ile (\%35.4) doğurtulmuştur. Perinatoloji kliniğinde takip edilen 14.902 gebenin (tüm doğumların \%26'sı) de 9215 tanesi sezaryen (\%61.8) ile doğurmuştur. Hastaneye yüksek oranda multipar başvurusu gerçekleşmektedir (\%39.2). Eski sezaryen tanılı hastalara \%99.8 oranında sezaryen uygulanmaktadır. Makat prezantasyon ile gelen gebelerin oranı beklenenden daha düşük olup, nullipar makat gelişlerde \%96.8 ve multipar makat gelişlerde \% 88.6'lik sezaryen oranı görülmüştür. Çoğul gebelik nedeniyle başvuran gebelerin oranı \%6.1 ve sezaryen oranı \%90.1 olarak saptanmıştır. Perinatoloji kliniğinde takip edilen başta preeklampsi olmak üzere farklı nedenlerle başvuran ve preterm doğum gerçekleştirilen gebelerin oranı $\% 25.1$ ve sezaryen oranı \%66.1 olarak gerçekleşmiştir.

Sonuç: Perinatoloji kliniği/tersiyer merkeze bağlı sezaryen oranları çok daha yüksek düzeydedir. Primer sezaryen oranlarını uygun düzeylerde tutmak için ısrarcı doğum indüksiyonu uygulamalarından kaçınmalı, tüp ligasyon işlemi için sezaryeni önermemeli ve uygun hastalarda makat gelişi durumlarında eksternal sefalik versiyon denenmelidir.

Anahtar sözcükler: Robson On Gruplu Sinıflandırma Sistemi, perinatoloji/tersiyer kliniği.

Correspondence: Ali Gedikbaşı, MD. Perinatology Department, Gynecology and Obstetrics Clinic, İstanbul Kanuni Sultan Süleyman Training and Research Hospital, İstanbul, Turkey. e-mail: alged_1971@yahoo.com / Received: August 15, 2019; Accepted: September 23, 2019

Please cite this article as: Kıyak H, Bolluk G, Canaz E, Yüksel S, Gedikbaşı A. The evaluation of cesarean section rates in accordance with Robson Ten-Group Classification System and the data of perinatology (tertiary center). Perinatal Journal 2019;27(2):89-100. doi:10.2399/prn.19.0272007 


\section{Introduction}

The cesarean section is defined as the delivery of fetus with the help of abdominal and uterine incisions, and its application is recommended only when there are increased morbidity and mortality risks for mother and/or fetus during vaginal delivery. ${ }^{[1,2]}$ The World Health Organization (WHO) indicated ideal rate of cesarean section as $10-15 \%$ in 1985 ; however, cesarean section is one of the most common surgical procedures in the world..$^{[3,4]}$ According to the data of the WHO, Turkey is among the countries which have the highest rates of cesarean section in the world, and the rates of cesarean section increased from 7\% to 53\% between 1993 and 2015..$^{[5,6]}$

Repeating cesarean section, dystocia, fetal distress and abnormal fetal presentation have been reported as the most common indications for cesarean section. ${ }^{[7]}$ Moreover, increased labor induction, the use of electronic fetal monitor becoming prevalent and the suboptimal evaluation, first labors being at more advanced ages, decreased practice of labor induction in preeclamptic pregnant women, increased use of assisted reproduction technologies and the multiple pregnancies accordingly, performing cesarean section in all breech presentation cases, not preferring vaginal delivery after cesarean section and the difficulty of follow-up, fear of vaginal delivery in young pregnant women as a social indication, decreased use of forceps and vacuum, the pressure created on obstetricians due to medicolegal issues that may arise in association with the operative labor are among the other reasons contributing to the increased rates of indications and cesarean section. ${ }^{[2,5,7-9]}$

With the proposal of the Turkish Ministry of Health, "Robson Ten-Group Classification System" (Robson's TGCS) has being used in obstetrics clinics since May $2012 .^{[10]}$ In this system, the women are classified in ten different groups based on 5 basic labor characteristics independent of each other: parity (nulliparous, multiparous, previous history of cesarean section), onset of the labor (spontaneous, induced, cesarean section before the labor), duration of pregnancy (preterm, term), number of fetus (singleton, multiple), and fetal presentation (head, breech, transverse). While this classification enables the simple, safe and clinically significant evaluation of every woman who admits for labor, it also makes it possible to inter- and intra-group comparisons of the rates of cesarean section. ${ }^{[11]}$ With the current version, Robson's TGCS is a clinical obstetric evaluation, and the groups are evaluated independent from each other.
On the other hand, when clinical experience and patient variety are evaluated together, differences can be observed among various hospitals and clinics, and it is also possible to see various clinical practices due to the different approaches of different clinics in the same department. Despite the idealized labor rates, the inclusion of high risk pregnancies in the patient cohort with the presence of a separate perinatology clinic within the department leads to the expectation of an increase in the rates of cesarean section. There are a limited number of literature findings on Robson data associated with different clinic and patient groups. Our purpose in this study is to evaluate the impact of differences between obstetrics clinics and perinatology clinics on Robson's TGCS as well as the data of obstetrics training clinic in our hospital which provides intensive service, and to see its reflection on labor outcomes.

\section{Methods}

Although Robson criteria have been recorded in our hospital since 2012 upon the instructions of the Ministry of Health, relevant data have been recorded regularly since 2015 after the digitalization of these records in 2014 and training the personnel who were responsible for recording procedures (Table 1). For the procurement of digital data, the pregnant women in the data system of the hos-

Table 1. Robson's Classification System.

\begin{tabular}{|ll}
\hline Groups & Description \\
\hline Group 1 & $\begin{array}{l}\text { Nulliparous women with a single cephalic pregnancy, } \geq 37 \\
\text { weeks of gestation in spontaneous labor }\end{array}$ \\
\hline Group 2 & $\begin{array}{l}\text { Nulliparous women with a single cephalic pregnancy at } \\
\text { term who either had labor induced or were delivered by } \\
\text { cesarean section before labor }\end{array}$ \\
\hline Group 3 & $\begin{array}{l}\text { Multiparous women without a previous uterine scar, with a } \\
\text { single cephalic pregnancy, } \geq 37 \text { weeks of gestation in } \\
\text { spontaneous labor }\end{array}$ \\
\hline Group 4 & $\begin{array}{l}\text { Multiparous women without a previous uterine scar, with a } \\
\text { single cephalic pregnancy, } \geq 37 \text { weeks of gestation who } \\
\text { either had labor induced or were delivered by cesarean } \\
\text { section before labor }\end{array}$ \\
\hline Group 5 & $\begin{array}{l}\text { Multiparous women with at least one previous uterine scar, } \\
\text { with a single cephalic pregnancy, } \geq 37 \text { weeks of gestation }\end{array}$ \\
\hline Group 6 & \begin{tabular}{l} 
Nulliparous women with a single breech pregnancy \\
\hline Group 7
\end{tabular} $\begin{array}{l}\text { Multiparous women with a single breech pregnancy, including } \\
\text { women with previous uterine scars }\end{array}$ \\
\hline Group 8 & $\begin{array}{l}\text { All multiple pregnancies, including those with previous } \\
\text { uterine scars }\end{array}$ \\
\hline Group 9 & $\begin{array}{l}\text { All women with a single pregnancy with a transverse or oblique } \\
\text { presentation, including women with previous uterine scars }\end{array}$ \\
\hline Group 10 & $\begin{array}{l}\text { All women with a single cephalic pregnancy, <37 weeks of } \\
\text { gestation, including women with previous scars }\end{array}$ \\
\hline
\end{tabular}


pital were classified in two groups according to the outpatient clinics that they were followed up and the clinic they were hospitalized (Pregnancy group and Perinatology group), and their clinic origins were reviewed. In this way, all data of obstetrics clinic, Pregnancy group and Perinatology group were obtained separately and in an aggregated form for each year (2015-2019).

This study is the retrospective review of the hospital data, and the records for the labors carried out in the obstetrics and perinatology clinics of the hospital between 01.01.2015 and 30.06.2019 were reviewed. The assessment of the study protocol and the approval were conducted by the local ethics committee of the hospital.

The WHO published Robson Classification: Implementation Manual in 2017, and provided some recommendations about the implementation. ${ }^{[12]}$ These recommendations about cesarean section and population data were applied to the sub-groups of Robson and given in Table 2 .

While problem-free pregnancies were followed up in obstetrics clinic, high risk pregnancies were followed up in perinatology outpatient clinics of the perinatology clinic and the perinatology service when necessary. However, the hospitalization for labor and the deliveries of all these patients were carried out in the same delivery rooms and operating rooms. Accordingly, the admission and hospitalization of pregnant women were conducted in two ways:

- The pregnant women who were monitored in pregnancy outpatient clinics and hospitalized after active labor or referred to the hospital for the first time under emergency conditions due to different reasons; the hospitalization of both groups are conducted through emergency obstetrics clinic.

- The pregnant women with perinatology record; they are the pregnant women for whom delivery is decided due to maternal or fetal indications when they are hospitalized in the perinatology unit or those followed up in perinatology outpatient clinic and delivered in the hospital in due course.

In line with the recommendations of the $\mathrm{WHO}$ and FIGO in 2016, the rates of the data were recalculated to see their contribution on their own weightings and the rates of cesarean section. ${ }^{[12,13]}$ In accordance with the recommendations, the groups, the numbers of cesarean section in the group, the total number of women who gave birth in the group, size of these groups compared to the available population, intra- group cesarean section rates, and actual and relative contributions of the group to the total rate of cesarean section were determined for the patients of Robson's TGCS in 7 separate columns. After the total data of obstetrics clinic were obtained, the data of the pregnancy group and perinatology group were also determined as indicated above. These data were evaluated for all patients of obstetrics clinic and pregnancy and perinatology groups in accordance with the recommendations and comments given in Table 2 .

Unfortunately, primary cesarean section rates, which are one of the data requested by the Ministry of Health, cannot be calculated by these tables established according to the Robson's TGCS. Since nulliparous and multiparous pregnancy groups are different than each other, both groups cannot be evaluated together in Robson's TGCS. We calculated them separately and presented their results.

We paid attention to the confidentiality of the personal data of the patients during pre-investigational study. In the data analysis, the qualitative variables were summarized in numbers and percentages.

\section{Results}

During the review of the data system, it was found that a total of 57,402 deliveries were carried out in İstanbul Kanuni Sultan Süleyman Training and Research Hospital between 01.01.2015 and 30.06.2019. In terms of the data record by years, a proper classification could not be made for 40 pregnant women in 2015, 4 pregnant women in 2016, 1 pregnant woman in 2017, and 2 pregnant women in 2018. The classification of these patients was not taken into consideration during the calculations. Similarly, it was reported that the failure of classification might occur. ${ }^{[12]}$

A total of 24,240 (42.2\%) out of 57,402 labors were carried out by cesarean section in the hospital during the relevant period. All data and cesarean section rates of the groups of entire obstetrics clinic are given by years in Table 3. After pregnancy clinic / outpatient clinic and perinatology clinic were distinguished, it was seen that $15,025(35.4 \%)$ of 42,500 deliveries ( $74 \%$ of all deliveries) in pregnancy outpatient clinic were carried out by cesarean section (the rates of relevant group were given in Table 4), and 9215 (61.8\%) of 14,902 pregnant women (26\% of all deliveries) who were hospitalized through perinatology clinic delivered by cesarean section (the rates of relevant group were given in Table 5). 
Table 2. WHO's Robson recommendations - Population interpretations and recommendations for the rates of cesarean section (Summarized and edited according to the reference 12).

\begin{tabular}{|c|c|c|c|}
\hline \multirow[b]{2}{*}{ Groups } & \multicolumn{2}{|c|}{ Type of population } & \multirow[b]{2}{*}{ CS recommendation (Column 5) } \\
\hline & Recommendation & Interpretation & \\
\hline $\begin{array}{l}\text { Group 1: nulliparous normal } \\
\text { labor, } \geq 37\end{array}$ & $\begin{array}{l}\text { It represents } 35-42 \% \\
\text { of the pregnancies }\end{array}$ & $\begin{array}{l}\text { A lower ratio indicates high } \\
\text { induction / cesarean section } \\
\text { before labor starts; it shows } \\
\text { increased rate of cesarean } \\
\text { section due to the presence } \\
\text { of high risk population }\end{array}$ & $\begin{array}{l}\text { Rates under } 10 \% \text { are achievable } \\
\text { This rate can be interpreted properly } \\
\text { when size rates of Groups } 1 \text { and } \\
2 \text { are considered. }\end{array}$ \\
\hline Group 2: nulliparous ind/cs & $\begin{array}{l}\text { The ratio of Group 1/ } \\
\text { Group } 2 \text { should be } 2: 1\end{array}$ & $\begin{array}{l}\text { If it is high, it means that we did } \\
\text { not induced sufficiently. }\end{array}$ & Consistently around $20-35 \%$ \\
\hline $\begin{array}{l}\text { Group 3: multiparous } \\
\text { normal labor, } \geq 37\end{array}$ & $\begin{array}{l}\text { It represents } 30 \% \text { of the } \\
\text { total pregnancies }\end{array}$ & $\begin{array}{l}\text { The reason for low rates of Groups } \\
3 \text { and } 4 \text { may be the high rates in } \\
\text { Group } 5 \text { and the accompanying } \\
\text { high rates of cesarean section. }\end{array}$ & $\begin{array}{l}\text { Not higher than } 3 \% \text { normally } \\
\text { In units with higher rates, this may } \\
\text { be due to poor data collection or } \\
\text { requesting tubal ligation. }\end{array}$ \\
\hline $\begin{array}{l}\text { Group 4: multiparous } \\
\text { ind/cs, } \geq 37\end{array}$ & $\begin{array}{l}\text { The ratio of Group 3/ } \\
\text { Group } 4 \text { should be higher } \\
\text { than the ratio of Group 1/ } \\
\text { Group } 2\end{array}$ & $\begin{array}{l}\text { A lower rate may indicate the } \\
\text { cesarean section before labor in } \\
\text { multiparous women, or the cesarean } \\
\text { section before labor due to tubal } \\
\text { ligation. }\end{array}$ & $\begin{array}{l}\text { It is rarely higher than } 15 \% \\
\text { A high rate of cesarean section in } \\
\text { Group } 4 \text { may reflect a high maternal } \\
\text { request for cesarean section even } \\
\text { if these women have delivered their } \\
\text { first pregnancy vaginally, or being } \\
\text { able to do tubal ligation. }\end{array}$ \\
\hline \multirow[t]{2}{*}{ Group 5: $\mathrm{cs}, \geq 37$} & \multirow[t]{2}{*}{$\begin{array}{l}\text { The size is roughly half of } \\
\text { the total cesarean section } \\
\text { rate in general }\end{array}$} & $\begin{array}{l}\text { If the Group is big, it means that } \\
\text { Groups } 1 \text { and } 2 \text { in particular have } \\
\text { high rates of cesarean section } \\
\text { in previous years. }\end{array}$ & $\begin{array}{l}\text { Rates of } 50-60 \% \text { are considered } \\
\text { appropriate provided you have } \\
\text { good maternal and perinatal } \\
\text { outcomes. }\end{array}$ \\
\hline & & $\begin{array}{l}\text { The size of this group may be }>15 \% \\
\text { in settings with high rates of } \\
\text { cesarean section. }\end{array}$ & $\begin{array}{l}\text { If the rates are higher, this is possibly } \\
\text { due to a larger group of women with } \\
2 \text { or more previous cesarean section } \\
\text { or due to a policy of scheduling } \\
\text { pre-labor cesarean section for all } \\
\text { women with } 1 \text { previous scar without } \\
\text { attempting a trial of labor. }\end{array}$ \\
\hline $\begin{array}{l}\text { Group 6: nulliparous breech } \\
\text { presentation }\end{array}$ & The total should be $3-4 \%$ & $\begin{array}{l}\text { If the total is over } 4 \% \text {, the reason is } \\
\text { usually a high rate of preterm deliveries } \\
\text { or a higher proportion of nulliparous } \\
\text { women. If the size of Group } 10 \text { is above } \\
4-5 \% \text {, then this hypothesis is correct. }\end{array}$ & \\
\hline $\begin{array}{l}\text { Group 7: multiparous breech } \\
\text { presentation, cs }\end{array}$ & $\begin{array}{l}\text { The ratio of Group } 6 / \text { Group } \\
7 \text { should be } 2: 1\end{array}$ & $\begin{array}{l}\text { If the rate is different, there is an } \\
\text { unexpected ratio of nulliparous/mu } \\
\text { Itiparous women. }\end{array}$ & \\
\hline $\begin{array}{l}\text { Group 8: multiple } \\
\text { pregnancy, cs }\end{array}$ & It should be $1.5-2 \%$ & $\begin{array}{l}\text { If it is higher, the hospital is probably } \\
\text { a tertiary center. } \\
\text { If it is lower, probably the majority of the } \\
\text { twin pregnancies twins are referred out }\end{array}$ & $\begin{array}{l}\text { It is usually around } 60 \% \text {. } \\
\text { The variations will depend on the type } \\
\text { of twin pregnancy, previous history } \\
\text { of cesarean section and the ratio } \\
\text { of nulliparous/multiparous women }\end{array}$ \\
\hline Group 9: Transverse, cs & It should be less than $1 \%$ & & $\begin{array}{l}\text { It should be } 100 \% \text {; if the woman } \\
\text { gives birth vaginally by internal version, } \\
\text { it should be classified as either cephalic } \\
\text { or breech presentation }\end{array}$ \\
\hline $\begin{array}{l}\text { Group 10: preterm labor, } \\
<37 \text {, cs }\end{array}$ & $\begin{array}{l}\text { It should be less than } 5 \% \\
\text { in normal risky settings }\end{array}$ & $\begin{array}{l}\text { If it is higher, the hospital is } \\
\text { probably a tertiary center. }\end{array}$ & $\begin{array}{l}\text { It is usually around } 30 \% \text { in the } \\
\text { most populations }\end{array}$ \\
\hline & & $\begin{array}{l}\text { If the rate of cesarean section is high, } \\
\text { it may suggest pre-labor cesarean } \\
\text { section due to fetal growth restriction } \\
\text { or preeclampsia and other complications. }\end{array}$ & $\begin{array}{l}\text { If it is higher than } 30 \% \text {, it is usually due } \\
\text { to the cases of high risk pregnancies } \\
\text { (e.g. fetal growth restriction, } \\
\text { preeclampsia) that will need preterm } \\
\text { pre-labor cesarean section }\end{array}$ \\
\hline
\end{tabular}

cs: Including previous cesarean section; ind: including those underwent induction; $\geq 37$ : 37 weeks of gestation and above; <37: less than 37 weeks of gestation 
The evaluation of cesarean section rates in accordance with Robson Ten-Group Classification System and the data of perinatology

Table 3. The labor data of all Obstetrics Clinic (Pregnancy Outpatient Clinics and Perinatology Clinic) according to the Robson's Ten-Group Classification System.

\begin{tabular}{|c|c|c|c|c|c|c|c|}
\hline \multirow[b]{2}{*}{ Year } & \multirow{2}{*}{$\begin{array}{l}\text { Column } 1 \\
\text { Robson } \\
\text { groups }\end{array}$} & \multirow{2}{*}{$\begin{array}{c}\text { Column } 2 \\
\text { CS } \\
\text { number } \\
\text { of group }\end{array}$} & \multirow{2}{*}{$\begin{array}{c}\text { Column } 3 \\
\text { Number of women } \\
\text { who delivered in } \\
\text { the group }\end{array}$} & \multirow{2}{*}{$\begin{array}{c}\text { Column } 4 \\
\text { Group size } \\
\text { according to the } \\
\text { population }(\%)\end{array}$} & \multirow{2}{*}{$\begin{array}{l}\text { Column } 5 \\
\text { Intra-group } \\
\text { CS rate (\%) }\end{array}$} & \multirow{2}{*}{$\begin{array}{c}\text { Column } 6 \\
\text { The actual contribution } \\
\text { of the group to the } \\
\text { total rate of CS (\%) }\end{array}$} & \multirow{2}{*}{$\begin{array}{c}\text { Column } 7 \\
\text { The relative contribution } \\
\text { of the group to the } \\
\text { total rate of CS (\%) }\end{array}$} \\
\hline & & & & & & & \\
\hline 2015 & $\begin{array}{c}1 \\
2 \\
3 \\
4 \\
5 \\
6 \\
7 \\
8 \\
9 \\
10 \\
\end{array}$ & $\begin{array}{c}579 \\
325 \\
619 \\
168 \\
2263 \\
147 \\
152 \\
302 \\
15 \\
1218 \\
\end{array}$ & $\begin{array}{c}2944 \\
542 \\
4810 \\
280 \\
2266 \\
150 \\
159 \\
336 \\
16 \\
2266 \\
\end{array}$ & $\begin{array}{c}21.4 \\
3.9 \\
34.9 \\
2.0 \\
16.6 \\
1.0 \\
1.1 \\
2.4 \\
0.1 \\
16.6 \\
\end{array}$ & $\begin{array}{l}19.7 \\
60.0 \\
12.9 \\
60.0 \\
99.9 \\
98.0 \\
95.6 \\
89.9 \\
93.8 \\
53.8\end{array}$ & $\begin{array}{c}4.1 \\
2.3 \\
4.4 \\
1.2 \\
16.4 \\
1.1 \\
1.1 \\
2.1 \\
0.1 \\
8.8 \\
\end{array}$ & $\begin{array}{c}10.1 \\
5.5 \\
10.7 \\
2.8 \\
39.5 \\
2.4 \\
2.5 \\
5.1 \\
0.2 \\
21.2 \\
\end{array}$ \\
\hline Total & & 5728 & 13,769 & 100.0 & 41.6 & 41.6 & 100.0 \\
\hline 2016 & $\begin{array}{c}1 \\
2 \\
3 \\
4 \\
5 \\
6 \\
7 \\
8 \\
9 \\
10 \\
\end{array}$ & $\begin{array}{c}778 \\
45 \\
665 \\
25 \\
2825 \\
143 \\
91 \\
310 \\
5 \\
1121 \\
\end{array}$ & $\begin{array}{c}3268 \\
188 \\
5150 \\
124 \\
2827 \\
151 \\
97 \\
357 \\
5 \\
2129 \\
\end{array}$ & $\begin{array}{c}22.9 \\
1.3 \\
36.0 \\
0.9 \\
19.8 \\
1.1 \\
0.7 \\
2.5 \\
0.1 \\
14.9 \\
\end{array}$ & $\begin{array}{c}23.8 \\
23.9 \\
12.9 \\
20.2 \\
99.9 \\
94.7 \\
93.8 \\
86.8 \\
100.0 \\
52.7 \\
\end{array}$ & $\begin{array}{c}5.4 \\
0.3 \\
4.7 \\
0.2 \\
19.8 \\
1.0 \\
0.6 \\
2.2 \\
0.0 \\
7.8 \\
\end{array}$ & $\begin{array}{c}12.9 \\
0.7 \\
11.1 \\
0.4 \\
47.0 \\
2.4 \\
1.5 \\
5.2 \\
0.1 \\
18.7 \\
\end{array}$ \\
\hline Total & & 6008 & 14,296 & 100.0 & 42.0 & 42.0 & 100.0 \\
\hline 2017 & $\begin{array}{c}1 \\
2 \\
3 \\
4 \\
5 \\
6 \\
7 \\
8 \\
9 \\
10 \\
\end{array}$ & $\begin{array}{c}536 \\
33 \\
612 \\
4 \\
2329 \\
78 \\
66 \\
273 \\
2 \\
1119 \\
\end{array}$ & $\begin{array}{c}2536 \\
67 \\
4988 \\
32 \\
2332 \\
83 \\
89 \\
335 \\
2 \\
1830 \\
\end{array}$ & $\begin{array}{c}20.6 \\
0.5 \\
40.6 \\
0.3 \\
19.0 \\
0.7 \\
0.7 \\
2.7 \\
0.1 \\
14.9\end{array}$ & $\begin{array}{c}21.1 \\
49.3 \\
12.3 \\
12.5 \\
99.9 \\
94.0 \\
74.2 \\
81.5 \\
100.0 \\
61.1\end{array}$ & $\begin{array}{c}4.4 \\
0.3 \\
5.0 \\
0.1 \\
18.9 \\
0.6 \\
0.5 \\
2.2 \\
0.0 \\
9.1\end{array}$ & $\begin{array}{c}10.6 \\
0.6 \\
12.1 \\
0.1 \\
46.1 \\
1.5 \\
1.3 \\
5.4 \\
0.1 \\
22.2 \\
\end{array}$ \\
\hline Total & & 5052 & 12,294 & 100.0 & 41.1 & 41.1 & 100.0 \\
\hline 2018 & $\begin{array}{c}1 \\
2 \\
3 \\
4 \\
5 \\
6 \\
7 \\
8 \\
9 \\
10 \\
\end{array}$ & $\begin{array}{c}668 \\
29 \\
504 \\
21 \\
2611 \\
117 \\
61 \\
291 \\
13 \\
655 \\
\end{array}$ & $\begin{array}{c}2456 \\
45 \\
4838 \\
52 \\
2619 \\
119 \\
71 \\
337 \\
13 \\
1225 \\
\end{array}$ & $\begin{array}{c}20.9 \\
0.4 \\
41.1 \\
0.4 \\
22.2 \\
1.0 \\
0.6 \\
2.9 \\
0.1 \\
10.4 \\
\end{array}$ & $\begin{array}{c}27.2 \\
64.4 \\
10.4 \\
40.4 \\
99.7 \\
98.3 \\
85.9 \\
86.4 \\
100.0 \\
53.5 \\
\end{array}$ & $\begin{array}{c}5.7 \\
0.2 \\
4.3 \\
0.2 \\
22.2 \\
1.0 \\
0.5 \\
2.5 \\
0.1 \\
5.6 \\
\end{array}$ & $\begin{array}{c}13.4 \\
0.6 \\
10.1 \\
0.4 \\
52.5 \\
2.4 \\
1.2 \\
5.9 \\
0.3 \\
13.2 \\
\end{array}$ \\
\hline Total & & 4970 & 11,775 & 100.0 & 42.2 & 42.2 & 100.0 \\
\hline 2019 & $\begin{array}{c}1 \\
2 \\
3 \\
4 \\
5 \\
6 \\
7 \\
8 \\
9 \\
10 \\
\end{array}$ & $\begin{array}{c}418 \\
1 \\
317 \\
0 \\
1225 \\
51 \\
36 \\
157 \\
9 \\
208 \\
\end{array}$ & $\begin{array}{c}1196 \\
7 \\
2193 \\
6 \\
1226 \\
51 \\
42 \\
175 \\
9 \\
363 \\
\end{array}$ & $\begin{array}{c}22.7 \\
0.1 \\
41.6 \\
0.1 \\
23.3 \\
1.0 \\
0.8 \\
3.3 \\
0.2 \\
6.9 \\
\end{array}$ & $\begin{array}{c}34.9 \\
14.3 \\
14.5 \\
0.0 \\
99.9 \\
100.0 \\
85.7 \\
89.7 \\
100.0 \\
57.3 \\
\end{array}$ & $\begin{array}{c}8.0 \\
0.0 \\
6.0 \\
0.0 \\
23.3 \\
1.0 \\
0.7 \\
3.0 \\
0.2 \\
3.9 \\
\end{array}$ & $\begin{array}{c}17.2 \\
0.0 \\
13.1 \\
0.0 \\
50.6 \\
2.1 \\
1.5 \\
6.5 \\
0.4 \\
8.6 \\
\end{array}$ \\
\hline Total & & 2422 & 5268 & 100.0 & 46.0 & 46.0 & 100.0 \\
\hline $\begin{array}{l}\text { The period } \\
\text { between } \\
01.01 .2015 \\
\text { and } \\
30.06 .2019\end{array}$ & $\begin{array}{c}1 \\
2 \\
3 \\
4 \\
5 \\
6 \\
7 \\
8 \\
9 \\
10 \\
\end{array}$ & $\begin{array}{c}2979 \\
433 \\
2717 \\
218 \\
11,253 \\
536 \\
406 \\
1333 \\
44 \\
4321 \\
\end{array}$ & $\begin{array}{c}12,400 \\
849 \\
21,979 \\
494 \\
11,270 \\
554 \\
458 \\
1540 \\
45 \\
7813 \\
\end{array}$ & $\begin{array}{c}21.6 \\
1.5 \\
38.3 \\
0.9 \\
19.6 \\
1.0 \\
0.8 \\
2.7 \\
0.1 \\
13.6 \\
\end{array}$ & $\begin{array}{l}24.0 \\
51.0 \\
12.4 \\
44.1 \\
99.8 \\
96.8 \\
88.6 \\
86.6 \\
97.8 \\
55.3 \\
\end{array}$ & $\begin{array}{c}5.2 \\
0.8 \\
4.7 \\
0.4 \\
19.6 \\
0.9 \\
0.7 \\
2.3 \\
0.1 \\
7.5 \\
\end{array}$ & $\begin{array}{c}12.3 \\
1.8 \\
11.2 \\
0.9 \\
46.4 \\
2.2 \\
1.7 \\
5.5 \\
0.2 \\
17.8 \\
\end{array}$ \\
\hline Total & & 24,240 & 57,402 & 100.0 & 42.2 & 42.2 & 100.0 \\
\hline
\end{tabular}


Table 4. The labor data of Pregnancy clinics according to the Robson's Ten-Group Classification System.

\begin{tabular}{|c|c|c|c|c|c|c|c|}
\hline & Column 1 & Column 2 & Column 3 & Column 4 & Column 5 & Column 6 & Column 7 \\
\hline Year & $\begin{array}{l}\text { Robson } \\
\text { groups }\end{array}$ & $\begin{array}{c}\text { CS } \\
\text { number } \\
\text { of group }\end{array}$ & $\begin{array}{l}\text { Number of women } \\
\text { who delivered } \\
\text { in the group }\end{array}$ & $\begin{array}{c}\text { Group size } \\
\text { according to the } \\
\text { population (\%) }\end{array}$ & $\begin{array}{l}\text { Intra-group } \\
\text { CS rate (\%) }\end{array}$ & $\begin{array}{l}\text { The actual contribution } \\
\text { of the group to the } \\
\text { total rate of CS (\%) }\end{array}$ & $\begin{array}{c}\text { The relative contribution } \\
\text { of the group to the } \\
\text { total rate of CS (\%) }\end{array}$ \\
\hline 2015 & $\begin{array}{c}1 \\
2 \\
3 \\
4 \\
5 \\
6 \\
7 \\
8 \\
9 \\
10\end{array}$ & $\begin{array}{c}380 \\
155 \\
377 \\
89 \\
1691 \\
109 \\
105 \\
90 \\
10 \\
586\end{array}$ & $\begin{array}{c}2430 \\
291 \\
4038 \\
122 \\
1694 \\
110 \\
112 \\
103 \\
11 \\
1319\end{array}$ & $\begin{array}{c}23.8 \\
2.8 \\
39.5 \\
1.2 \\
16.6 \\
1.1 \\
1.1 \\
1.0 \\
0.1 \\
12.9\end{array}$ & $\begin{array}{c}15.6 \\
53.3 \\
9.3 \\
73.0 \\
99.8 \\
99.1 \\
93.8 \\
87.4 \\
90.9 \\
44.4\end{array}$ & $\begin{array}{c}3.8 \\
1.5 \\
3.7 \\
0.9 \\
16.5 \\
1.1 \\
1.0 \\
0.9 \\
0.1 \\
5.7\end{array}$ & $\begin{array}{c}10.6 \\
4.3 \\
10.5 \\
2.5 \\
47.1 \\
3.0 \\
2.9 \\
2.5 \\
0.3 \\
16.3\end{array}$ \\
\hline Total & & 3592 & 10,230 & 100.0 & 35.1 & 35.1 & 100.0 \\
\hline 2016 & $\begin{array}{c}1 \\
2 \\
2 \\
3 \\
4 \\
5 \\
6 \\
7 \\
8 \\
9 \\
10\end{array}$ & $\begin{array}{c}519 \\
16 \\
427 \\
8 \\
2183 \\
84 \\
58 \\
145 \\
4 \\
498\end{array}$ & $\begin{array}{c}2762 \\
99 \\
4436 \\
61 \\
2185 \\
88 \\
63 \\
174 \\
4 \\
1172\end{array}$ & $\begin{array}{c}25.0 \\
0.9 \\
40.2 \\
0.6 \\
19.8 \\
0.8 \\
0.6 \\
1.6 \\
0.0 \\
10.6\end{array}$ & $\begin{array}{c}18.8 \\
16.2 \\
9.6 \\
13.1 \\
99.9 \\
95.5 \\
92.1 \\
83.3 \\
100.0 \\
42.5\end{array}$ & $\begin{array}{c}4.7 \\
0.1 \\
3.9 \\
0.1 \\
19.8 \\
0.8 \\
0.5 \\
1.3 \\
0.0 \\
4.6\end{array}$ & $\begin{array}{c}13.2 \\
0.4 \\
10.8 \\
0.2 \\
55.4 \\
2.1 \\
1.5 \\
3.7 \\
0.1 \\
12.6\end{array}$ \\
\hline Total & & 3942 & 11,044 & 100.0 & 35.7 & 35.7 & 100.0 \\
\hline 2017 & $\begin{array}{c}1 \\
2 \\
3 \\
4 \\
5 \\
6 \\
7 \\
7 \\
8 \\
9 \\
10 \\
\end{array}$ & $\begin{array}{c}334 \\
12 \\
381 \\
1 \\
1741 \\
48 \\
50 \\
102 \\
2 \\
475 \\
\end{array}$ & $\begin{array}{c}2045 \\
25 \\
4145 \\
7 \\
1744 \\
50 \\
69 \\
140 \\
2 \\
905\end{array}$ & $\begin{array}{c}22.4 \\
0.3 \\
45.4 \\
0.1 \\
19.1 \\
0.5 \\
0.8 \\
1.5 \\
0.0 \\
9.9\end{array}$ & $\begin{array}{c}16.3 \\
48.0 \\
9.2 \\
0.1 \\
99.8 \\
96.0 \\
72.5 \\
72.9 \\
100.0 \\
52.5\end{array}$ & $\begin{array}{c}3.6 \\
0.1 \\
4.1 \\
0.0 \\
19.1 \\
0.5 \\
0.5 \\
1.1 \\
0.2 \\
5.2\end{array}$ & $\begin{array}{c}10.6 \\
0.4 \\
12.1 \\
0.0 \\
55.4 \\
1.5 \\
1.6 \\
3.3 \\
0.0 \\
15.1\end{array}$ \\
\hline Total & & 3146 & 9132 & 100.0 & 34.5 & 34.5 & 100.0 \\
\hline 2018 & $\begin{array}{c}1 \\
2 \\
3 \\
4 \\
5 \\
6 \\
7 \\
8 \\
9 \\
10\end{array}$ & $\begin{array}{c}391 \\
20 \\
303 \\
15 \\
1763 \\
62 \\
46 \\
115 \\
8 \\
233\end{array}$ & $\begin{array}{c}1818 \\
31 \\
3951 \\
37 \\
1771 \\
63 \\
52 \\
143 \\
8 \\
538\end{array}$ & $\begin{array}{c}21.7 \\
0.4 \\
47.0 \\
0.4 \\
21.1 \\
0.7 \\
0.6 \\
1.7 \\
0.1 \\
6.4\end{array}$ & $\begin{array}{c}21.5 \\
64.5 \\
7.7 \\
40.5 \\
99.5 \\
98.4 \\
88.5 \\
80.4 \\
100.0 \\
43.3\end{array}$ & $\begin{array}{c}4.6 \\
0.2 \\
3.6 \\
0.2 \\
21.0 \\
0.7 \\
0.5 \\
1.4 \\
0.1 \\
2.8\end{array}$ & $\begin{array}{c}13.2 \\
0.7 \\
10.3 \\
0.5 \\
59.6 \\
2.1 \\
1.6 \\
3.9 \\
0.3 \\
7.8\end{array}$ \\
\hline Total & & 2956 & 8412 & 100.0 & 35.1 & 35.1 & 100.0 \\
\hline 2019 & $\begin{array}{c}1 \\
2 \\
3 \\
4 \\
5 \\
6 \\
7 \\
8 \\
9 \\
10 \\
\end{array}$ & $\begin{array}{c}224 \\
1 \\
178 \\
0 \\
802 \\
30 \\
24 \\
65 \\
8 \\
57\end{array}$ & $\begin{array}{c}849 \\
6 \\
1742 \\
2 \\
803 \\
30 \\
28 \\
74 \\
8 \\
140\end{array}$ & $\begin{array}{c}23.1 \\
0.2 \\
47.3 \\
0.1 \\
21.8 \\
0.8 \\
0.8 \\
2.0 \\
0.2 \\
3.8\end{array}$ & $\begin{array}{c}26.4 \\
16.7 \\
10.2 \\
0.0 \\
99.9 \\
100.0 \\
85.7 \\
87.8 \\
100.0 \\
40.7\end{array}$ & $\begin{array}{c}6.1 \\
0.0 \\
4.8 \\
0.0 \\
21.8 \\
0.8 \\
0.7 \\
1.8 \\
0.2 \\
1.5\end{array}$ & $\begin{array}{c}16.1 \\
0.0 \\
12.8 \\
0.0 \\
57.7 \\
2.2 \\
1.8 \\
4.7 \\
0.6 \\
4.1\end{array}$ \\
\hline Total & & 1389 & 3682 & 100.0 & 37.7 & 37.7 & 100.0 \\
\hline $\begin{array}{l}\text { The period } \\
\text { between } \\
01.01 .2015 \\
\text { and } \\
30.06 .2019\end{array}$ & $\begin{array}{c}1 \\
2 \\
3 \\
4 \\
5 \\
6 \\
7 \\
8 \\
9 \\
10\end{array}$ & $\begin{array}{c}1848 \\
204 \\
1666 \\
113 \\
8180 \\
333 \\
283 \\
517 \\
32 \\
1849\end{array}$ & $\begin{array}{c}9904 \\
452 \\
18,312 \\
229 \\
8197 \\
341 \\
324 \\
634 \\
33 \\
4074\end{array}$ & $\begin{array}{c}23.3 \\
1.1 \\
43.1 \\
0.5 \\
19.3 \\
0.8 \\
0.8 \\
1.5 \\
0.1 \\
9.6\end{array}$ & $\begin{array}{c}18.7 \\
45.1 \\
9.1 \\
49.3 \\
99.8 \\
97.7 \\
87.3 \\
81.5 \\
97.0 \\
45.4\end{array}$ & $\begin{array}{l}4.4 \\
0.5 \\
3.9 \\
0.3 \\
19.2 \\
0.8 \\
0.7 \\
1.2 \\
0.1 \\
4.4 \\
\end{array}$ & $\begin{array}{c}12.3 \\
1.4 \\
11.1 \\
0.8 \\
54.4 \\
2.2 \\
1.9 \\
3.4 \\
0.2 \\
12.3\end{array}$ \\
\hline Total & & 15,025 & 42,500 & 100.0 & 35.4 & 35.4 & 100.0 \\
\hline
\end{tabular}


The evaluation of cesarean section rates in accordance with Robson Ten-Group Classification System and the data of perinatology

Tablo 5. The labor data of Perinatology patients according to the Robson's Ten-Group Classification System.

\begin{tabular}{|c|c|c|c|c|c|c|c|}
\hline & Column 1 & Column 2 & Column 3 & Column 4 & Column 5 & Column 6 & Column 7 \\
\hline Year & $\begin{array}{l}\text { Robson } \\
\text { groups }\end{array}$ & $\begin{array}{c}\text { CS } \\
\text { number } \\
\text { of group }\end{array}$ & $\begin{array}{l}\text { Number of women } \\
\text { who delivered } \\
\text { in the group }\end{array}$ & $\begin{array}{l}\text { Group size } \\
\text { according to the } \\
\text { population (\%) }\end{array}$ & $\begin{array}{l}\text { Intra-group } \\
\text { CS rate (\%) }\end{array}$ & $\begin{array}{l}\text { The actual contribution } \\
\text { of the group to the } \\
\text { total rate of CS (\%) }\end{array}$ & $\begin{array}{c}\text { The relative contribution } \\
\text { of the group to the } \\
\text { total rate of CS (\%) }\end{array}$ \\
\hline 2015 & $\begin{array}{c}1 \\
2 \\
3 \\
4 \\
5 \\
6 \\
7 \\
8 \\
9 \\
10\end{array}$ & $\begin{array}{c}199 \\
170 \\
242 \\
79 \\
572 \\
38 \\
47 \\
212 \\
5 \\
632\end{array}$ & $\begin{array}{c}514 \\
251 \\
772 \\
158 \\
572 \\
40 \\
47 \\
233 \\
5 \\
947\end{array}$ & $\begin{array}{c}14.5 \\
7.1 \\
21.8 \\
4.5 \\
16.2 \\
1.1 \\
1.3 \\
6.6 \\
0.1 \\
26.8\end{array}$ & $\begin{array}{c}38.7 \\
67.7 \\
31.3 \\
50.0 \\
100.0 \\
95.0 \\
100.0 \\
91.0 \\
100.0 \\
66.7\end{array}$ & $\begin{array}{c}5.7 \\
4.8 \\
6.8 \\
2.2 \\
16.2 \\
1.1 \\
1.3 \\
6.0 \\
0.1 \\
17.9\end{array}$ & $\begin{array}{c}9.1 \\
7.7 \\
11.0 \\
3.6 \\
26.1 \\
1.7 \\
2.1 \\
9.7 \\
0.2 \\
28.8\end{array}$ \\
\hline Total & & 2196 & 3539 & 100.0 & 62.1 & 62.1 & 100.0 \\
\hline 2016 & $\begin{array}{c}1 \\
2 \\
2 \\
3 \\
4 \\
5 \\
6 \\
7 \\
7 \\
8 \\
9 \\
10\end{array}$ & $\begin{array}{c}259 \\
29 \\
238 \\
17 \\
642 \\
59 \\
33 \\
165 \\
1 \\
623\end{array}$ & $\begin{array}{c}506 \\
89 \\
714 \\
63 \\
642 \\
63 \\
34 \\
183 \\
1 \\
957 \\
\end{array}$ & $\begin{array}{c}15.6 \\
2.8 \\
22.0 \\
1.9 \\
19.7 \\
1.9 \\
1.0 \\
5.6 \\
0.0 \\
29.4\end{array}$ & $\begin{array}{c}51.2 \\
32.6 \\
33.3 \\
27.0 \\
100.0 \\
93.7 \\
97.1 \\
90.2 \\
100.0 \\
65.1\end{array}$ & $\begin{array}{c}8.0 \\
0.9 \\
7.3 \\
0.5 \\
19.7 \\
1.8 \\
1.0 \\
5.1 \\
0.0 \\
19.2\end{array}$ & $\begin{array}{c}12.5 \\
1.4 \\
11.5 \\
0.8 \\
31.1 \\
2.9 \\
1.6 \\
8.0 \\
0.0 \\
30.2\end{array}$ \\
\hline Total & & 2066 & 3252 & 100.0 & 63.5 & 63.5 & 100.0 \\
\hline 2017 & $\begin{array}{c}1 \\
2 \\
3 \\
4 \\
5 \\
6 \\
7 \\
7 \\
8 \\
9 \\
10 \\
\end{array}$ & $\begin{array}{c}202 \\
21 \\
231 \\
3 \\
588 \\
30 \\
16 \\
171 \\
0 \\
644\end{array}$ & $\begin{array}{c}491 \\
42 \\
843 \\
25 \\
588 \\
33 \\
20 \\
195 \\
0 \\
925 \\
\end{array}$ & $\begin{array}{c}15.5 \\
1.3 \\
26.7 \\
0.8 \\
18.6 \\
1.0 \\
0.6 \\
6.2 \\
0.0 \\
29.3\end{array}$ & $\begin{array}{c}41.1 \\
50.0 \\
27.4 \\
12.0 \\
100.0 \\
90.9 \\
80.0 \\
87.7 \\
0.0 \\
69.6\end{array}$ & $\begin{array}{c}6.4 \\
0.7 \\
7.3 \\
0.1 \\
18.6 \\
0.9 \\
0.5 \\
5.5 \\
0.0 \\
20.4 \\
\end{array}$ & $\begin{array}{c}10.6 \\
1.1 \\
12.1 \\
0.2 \\
30.8 \\
1.6 \\
0.8 \\
9.0 \\
0.0 \\
33.8\end{array}$ \\
\hline Total & & 1906 & 3162 & 100.0 & 60.3 & 60.3 & 100.0 \\
\hline 2018 & $\begin{array}{c}1 \\
2 \\
3 \\
4 \\
5 \\
6 \\
7 \\
8 \\
9 \\
10 \\
\end{array}$ & $\begin{array}{c}277 \\
9 \\
201 \\
6 \\
848 \\
55 \\
15 \\
176 \\
5 \\
422 \\
\end{array}$ & $\begin{array}{c}638 \\
14 \\
887 \\
15 \\
848 \\
56 \\
19 \\
194 \\
5 \\
687\end{array}$ & $\begin{array}{c}19.0 \\
0.4 \\
26.4 \\
0.4 \\
25.3 \\
1.7 \\
0.6 \\
5.8 \\
0.1 \\
20.4\end{array}$ & $\begin{array}{c}43.4 \\
64.3 \\
22.7 \\
40.0 \\
100.0 \\
98.2 \\
78.9 \\
90.7 \\
100.0 \\
61.4\end{array}$ & $\begin{array}{c}8.2 \\
0.3 \\
6.0 \\
0.2 \\
25.2 \\
1.6 \\
0.4 \\
5.2 \\
0.1 \\
12.6\end{array}$ & $\begin{array}{c}13.8 \\
0.4 \\
10.0 \\
0.3 \\
42.1 \\
2.7 \\
0.7 \\
8.8 \\
0.2 \\
21.0\end{array}$ \\
\hline Total & & 2014 & 3363 & 100.0 & 59.9 & 59.9 & 100.0 \\
\hline 2019 & $\begin{array}{c}1 \\
2 \\
3 \\
4 \\
5 \\
6 \\
7 \\
8 \\
9 \\
10 \\
\end{array}$ & $\begin{array}{c}194 \\
0 \\
139 \\
0 \\
423 \\
21 \\
12 \\
92 \\
1 \\
151\end{array}$ & $\begin{array}{c}347 \\
1 \\
451 \\
4 \\
423 \\
21 \\
14 \\
101 \\
1 \\
223 \\
\end{array}$ & $\begin{array}{c}21.9 \\
0.1 \\
28.4 \\
0.3 \\
26.7 \\
1.3 \\
0.9 \\
6.4 \\
0.1 \\
14.1\end{array}$ & $\begin{array}{c}55.9 \\
0.0 \\
30.8 \\
0.0 \\
100.0 \\
100.0 \\
85.7 \\
91.1 \\
100.0 \\
67.7\end{array}$ & $\begin{array}{c}12.2 \\
0.0 \\
8.8 \\
0.0 \\
26.7 \\
1.3 \\
0.8 \\
5.8 \\
0.1 \\
9.5\end{array}$ & $\begin{array}{c}18.8 \\
0.0 \\
13.5 \\
0.0 \\
40.9 \\
2.0 \\
1.2 \\
8.9 \\
0.1 \\
14.6\end{array}$ \\
\hline Total & & 1033 & 1586 & 100.0 & 65.1 & 65.1 & 100.0 \\
\hline $\begin{array}{l}\text { The period } \\
\text { between } \\
01.01 .2015 \\
\text { and } \\
30.06 .2019\end{array}$ & $\begin{array}{c}1 \\
2 \\
3 \\
4 \\
5 \\
6 \\
7 \\
8 \\
9 \\
10 \\
\end{array}$ & $\begin{array}{c}1131 \\
229 \\
1051 \\
105 \\
3073 \\
203 \\
123 \\
816 \\
12 \\
2472 \\
\end{array}$ & $\begin{array}{c}2496 \\
397 \\
3667 \\
265 \\
3073 \\
213 \\
134 \\
906 \\
12 \\
3739 \\
\end{array}$ & $\begin{array}{c}16.8 \\
2.7 \\
24.6 \\
1.8 \\
20.6 \\
1.4 \\
0.9 \\
6.1 \\
0.1 \\
25.1 \\
\end{array}$ & $\begin{array}{c}45.3 \\
57.7 \\
28.7 \\
39.6 \\
100.0 \\
95.3 \\
91.8 \\
90.1 \\
100.0 \\
66.1 \\
\end{array}$ & $\begin{array}{c}7.6 \\
1.5 \\
7.1 \\
0.7 \\
20.6 \\
1.4 \\
0.8 \\
5.5 \\
0.1 \\
16.6 \\
\end{array}$ & $\begin{array}{c}12.3 \\
2.5 \\
11.4 \\
1.1 \\
33.3 \\
2.2 \\
1.3 \\
8.9 \\
0.1 \\
26.9 \\
\end{array}$ \\
\hline Total & & 9215 & 14,902 & 100.0 & 61.8 & 61.8 & 100.0 \\
\hline
\end{tabular}


These total data for 4.5 years were evaluated separately and in an aggregated form, and the data and calculated rates of total, Pregnancy group and Perinatology Group of the obstetrics clinic were compared with the summarized rates and the data given in Table $\mathbf{2}$ for the WHO's Robson's TGCS (Table 6).

However, the primary cesarean section rates (performing cesarean section to pregnant women for the first time regardless of nulliparous or multiparous pregnancy), which are requested from the hospitals regularly by the Turkish Health Ministry, cannot be calculated according to the Robson's TGCS. According to the data obtained from the statistics unit of the hospital for the years 2015, 2016, 2017, 2018 and first 6 months of 2019, the primary cesarean section rates of the hospital were $20.1 \%, 19.0 \%, 16.2 \%, 16.7 \%$ and $20.0 \%$, respectively.

As seen in Table 6, it was found that more multiparous pregnant women admitted to the hospital than the expected rates of nulliparous/multiparous pregnant women. It is seen for each group that the rates of Group 1 and Group 2 involving nulliparous pregnancies are highly above the ratio of 2:1 by years. The results of the cesarean section rates in Group 1 and Group 2 for all pregnant women of Obstetrics clinic, Pregnancy clinic and Perinatology clinic were $24.0 \%$ and $51.0 \%, 18.7 \%$ and $45.1 \%$, and $45.3 \%$ and $57.7 \%$, respectively.

It is seen in the Obstetrics and Pregnancy groups that the rates of Group 3 and Group 4 patients involving multigravida pregnancies are higher than the expected. On the other hand, the admission rate of $26.4 \%$ was achieved in the Perinatology group, which is below the total rate of $30 \%$ expected from these two groups. It was seen in all groups that the cesarean rates were highly above the targeted rates which were 3\% for Group 3 and $15 \%$ for Group 4.

It is understood through the data that the rates of patients who constitute Group 5 and had cesarean section or uterine incision previously are about $19-20 \%$ for all groups and that the cesarean section was performed in almost all pregnant women who admitted with the history of previous cesarean section.

However, the admission rates of Group 6 constituted by breech presentation cases and involving nulliparous cases and Group 7 involving multiparous cases were far below the recommended rate of $3-4 \%$ and the ratio of these groups was lower than 2:1. It was found that the rates of cesarean section were $96.8 \%, 97.7 \%$ and $95.3 \%$

Table 6. The results of Obstetrics, Pregnancy and Perinatology groups according to the recommendations.

\begin{tabular}{|c|c|c|c|c|c|}
\hline Criteria & & $\begin{array}{c}\text { WHO } \\
\text { recommendations }\end{array}$ & $\begin{array}{l}\text { Total data of } \\
\text { Obstetrics Clinic }\end{array}$ & $\begin{array}{l}\text { Pregnancy } \\
\text { clinic }\end{array}$ & $\begin{array}{l}\text { Perinatology } \\
\text { clinic }\end{array}$ \\
\hline \multirow[t]{2}{*}{ Group 1 / Group 2} & Total & $35-42 \%$ & $23.1 \%$ & $24.4 \%$ & $19.4 \%$ \\
\hline & Ratio & $2: 1$ & 14.6 & 21.9 & 6.3 \\
\hline Group 1 & CS rate & $10 \%$ & $24.0 \%$ & $18.7 \%$ & $45.3 \%$ \\
\hline Group 2 & CS rate & $20-35 \%$ & $51.0 \%$ & $45.1 \%$ & $57.7 \%$ \\
\hline \multirow[t]{2}{*}{ Group 3 / Group 4} & Total & $30 \%$ & $39.2 \%$ & $43.6 \%$ & $26.4 \%$ \\
\hline & Ratio & $>2: 1$ & 44.5 & 80 & 13.8 \\
\hline Group 3 & CS rate & $<3 \%$ & $12.4 \%$ & $9.1 \%$ & $28.7 \%$ \\
\hline Group 4 & CS rate & $<15 \%$ & $44.1 \%$ & $49.3 \%$ & $39.6 \%$ \\
\hline \multirow[t]{2}{*}{ Group 5} & Size & $15 \%$ & $19.6 \%$ & $19.3 \%$ & $20.6 \%$ \\
\hline & CS rate & $50-60 \%$ & $99.8 \%$ & $99.8 \%$ & $100 \%$ \\
\hline \multirow[t]{2}{*}{ Group 6 / Group 7} & Total & $3-4 \%$ & $1.8 \%$ & $1.6 \%$ & $2.3 \%$ \\
\hline & Ratio & $2: 1$ & 1.2 & 1.1 & 1.6 \\
\hline Group 6 & CS rate & $4 \%$ & $96.8 \%$ & $97.7 \%$ & $95.3 \%$ \\
\hline Group 7 & CS rate & $4 \%$ & $88.6 \%$ & $87.3 \%$ & $91.8 \%$ \\
\hline \multirow[t]{2}{*}{ Group 8} & Size & $1.5-2 \%$ & $2.7 \%$ & $1.5 \%$ & $6.1 \%$ \\
\hline & CS rate & $60 \%$ & $86.6 \%$ & $81.5 \%$ & $90.1 \%$ \\
\hline \multirow[t]{2}{*}{ Group 9} & Size & $<1 \%$ & $0.1 \%$ & $0.1 \%$ & $0.1 \%$ \\
\hline & CS rate & $100 \%$ & $97.8 \%$ & $97.0 \%$ & $100 \%$ \\
\hline \multirow[t]{2}{*}{ Group 10} & Size & $<5 \%$ & $13.6 \%$ & $9.6 \%$ & $25.1 \%$ \\
\hline & CS rate & $30 \%$ & $55.3 \%$ & $45.4 \%$ & $66.1 \%$ \\
\hline
\end{tabular}


for Group 6 and 88.6\%, 87.3\% and 91.8\% for Group 7, respectively.

Group 8 involves multiple pregnancies and the rates in Pregnancy group are consistent with those recommended by the WHO; however, it is seen that the rate of $6.1 \%$ in the Perinatology group impacts the total rate and total group rate is $2.7 \%$. Again, all groups have higher rates of cesarean section than the desired cesarean section rate of $60 \%$, and it was $86.6 \%, 81.5 \%$ and $90.1 \%$ for Obstetrics, Pregnancy and Perinatology groups, respectively.

The rates of cesarean section for all three groups were found $97.8 \%, 97 \%$ and $100 \%$ due to the rates given in the data of 2015, which was the first year when Group 9 was evaluated in terms of transverse presentation. The results are as they should be except the data of 2015, and all rates of other years are $100 \%$.

Although targeted group size was $<5 \%$ in Group 10 which has admission difference due to high risk pregnancies and in which preterm pregnant women that cause deviations from the recommendations of the WHO in general are evaluated, the admission rates for Obstetrics, Pregnancy and Perinatology groups were 13.6\%, 9.6\% and $25.1 \%$, respectively. Also, the rates of $55.3 \%, 45.4 \%$ and $66.1 \%$ were found for these groups which were highly above the targeted rate of $30 \%$ for cesarean section. With these data, it was seen that the hospital does not only serve to the pregnant women who need to have preterm labor through Perinatology clinic, but also through the delivery room where many pregnant women need to have preterm labor due to various reasons.

\section{Discussion}

Robson's TGCS helps to classify every pregnant woman who admits for labor in one of the 10 groups which are completely inclusive in a way not involving in two different but correct conditions simultaneously. This uniform coding system provides a strict quality control system for complications by determining specific and diagnostic criteria for the complications. ${ }^{[12]}$ Our study is the second study in Turkey about a tertiary center. ${ }^{[14]}$ Yet, in our literature review, we found a limited number of data on the use of Robson's TGCS in the tertiary centers in the world. ${ }^{[15-22]}$ We found only 2 of these studies ${ }^{[15,16]}$ that the labor data between tertiary centers and secondary delivery units were evaluated in terms of Robson's TGCS.
The study data and the relevant results are affected highly by the population and clinical variety of the pregnant women who admitted to the hospital. Therefore, a follow-up systematic has been developed by a perinatology clinic or tertiary center for high risk pregnancies in terms of both fetal and maternal aspects. In this way, the need of finalizing pregnancy by vaginal delivery as a natural method or by cesarean section due to compulsory reasons varies depending on the clinical condition and it is seen that cesarean section is performed more frequently in tertiary centers having perinatology units. ${ }^{[15,16]}$ The perinatology unit of our hospital serves as an intensive care unit, and approximately one fourth of all cases $(26 \%)$ are treated and deliver in this unit. When we reviewed the study results, we found differences among the groups in terms of Robson's TGCS; the general rate of cesarean section in the perinatology unit of our hospital was $61.8 \%$ while it was $35.4 \%$ in the Pregnancy group, and the mean of our total clinic data which was the common result of these two groups was $42.2 \%$.

We also found that the numbers and rates of nulliparous and multiparous pregnant women who admitted to the hospital were different than the values provided by the WHO. While the mean values given by the $\mathrm{WHO}$ as example were $35-42 \%$ for primigravida cases and $30 \%$ for multigravida cases, they were quite the opposite for our hospital data, and it was $23.1 \%$ for primigravida cases and $39.2 \%$ for multigravida cases. In the sub-group analysis, the mean admission rates for primigravida and multigravida cases were $24.4 \%$ and $43.6 \%$ in the Pregnancy group and $19.4 \%$ and $26.4 \%$ in the Perinatology group, respectively. With these rates, the rate of multigravida patients admitting to the perinatology unit is at an acceptable level. However, in two previous studies evaluating Robson's TGCS data in Turkey reported that the rates were consistent with those recommended by the WHO. ${ }^{[14,23]}$ In this respect, it can be thought that many primigravida pregnant women would like to have their labors in private centers due to many social factors in Turkey or in the region that the hospital is located in a narrow perspective ${ }^{[24,25]}$ However, it is seen that the rates of cesarean section are high in Groups 1-4 which involve primigravida and multigravida cases. WHO motions report that the decision for cesarean section instead of induction is made more easily in primigravida cases, and therefore induction procedures should be focused on much more, and the decision for cesarean section is made more easily in multigravida 
cases due to the families' request of tubal ligation. ${ }^{[12]}$ The WHO recommends evaluating the relative contributions of Groups 1, 2 and 5 to general rate of cesarean section, because these three groups are responsible for $2 / 3(66 \%)$ of the cesarean section procedures in hospitals, and the focus should be on these three groups for the efforts of decreasing general rate of cesarean section in hospitals. In fact, the higher the general rate of cesarean rate is, the more focus should be on Group 1. ${ }^{[26]}$

The group of pregnant women with the history of previous cesarean section (Group 5) is the group which is an opportunity to decrease the rates of cesarean section in this regard. From this aspect, the WHO predicts the rate of cesarean section $50-60 \%$ in a group with a size of $15 \%$ approximately. However, some factors such as the number of previous cesarean section, the type of uterine incision performed previously, week of gestation, the presence of multiple pregnancy and maternal obesity are the significant limitations for the vaginal labor after cesarean section. Taking these factors into consideration and following up the labor under appropriate conditions and in the environments prepared in advance are the important necessities. ${ }^{[27]}$ With regard to our study, the lack of relevant practices in the obstetrics unit and delivery room can be expressed as a significant issue; however, it is important that the patients have appropriate conditions and the pregnant women are willing to participate. Besides, a majority of the pregnant women who admit to the hospital having two or more cesarean section and the hospital being a referral center for placenta previa and placenta percreta cases can be shown as the reasons for the cesarean section rate of 99\% for Pregnancy group and 100\% for Perinatology group which are 19-20\% in Group 5.

Interestingly, in consistent with our data, two other studies from Turkey ${ }^{[1,23]}$ reported the admission rates of pregnancies with breech presentation 3-4\% lower than the recommended rates. Group 9, which had transverse and oblique presentations, had similar low rate. Cochrane reviews report that the pregnancies with breech presentation have lower rates of perinatal/neonatal mortality and morbidity by cesarean section. ${ }^{[28]}$ This may explain the low rate of admission of the patients in this group; pregnant women with breech presentation are delivered by a planned labor decision. ${ }^{[25]}$ However, the societies in many countries recommend vaginal delivery or cesarean section for the pregnancies with breech presentation after trying external cephalic ver- sion. ${ }^{[29]}$ Eliminating the lack of this practice by trainings through the societies can be a potential precaution.

In the hospital which is a reference center for multiple pregnancies constituting Group 8 and for Group 10 involving preterm labors due to gestational complications, the mean rate of hospital is $2.7 \%$ and it is $6.1 \%$ in the perinatology unit for multiple pregnancies, and they are $13.5 \%$ and $25.1 \%$ for preterm labors, respectively, which are higher than the rates recommended by the WHO. Similar high rates in both groups are also reported by other studies evaluating tertiary centers. ${ }^{[1,1,2,21]}$ However, we associated the high rates of Group 8 and Group 10 with the rates of primary cesarean section which involve the cases with previous cesarean section and already high.

One of the limitations of our study is shift order in our hospital. Delivery room is a common area, and Pregnancy and Perinatology groups do not have separate delivery rooms. One of the reasons for the high rates in the pregnancy group is considered that the perinatology teams and experts working in the delivery room under shift conditions provide direct service to emergency pregnant women with high risk who admit to the hospital for the first time without any perinatology record and have their labors under emergency conditions. Besides, the lack of performance criteria according to the rates of cesarean section when providing delivery service is also important. ${ }^{[30]}$ Carrying out cesarean section at high rates in pregnant women who are evaluated to be in the high risk pregnancy group in line with the data and the literature is an expected condition.

\section{Conclusion}

It is important to evaluate the services provided by the hospital on different aspects and analyze the data accordingly. Therefore, except general obstetric data, the admitting patients and their results should be distinguished in terms of the services of tertiary center, and perinatology results also should be presented except general pregnancy data. The rate of cesarean section was $61.8 \%$ in the Perinatology group which contain all groups and $35.4 \%$ in the Pregnancy group.

In the studies for decreasing the hospital rates of cesarean section, it is important to do different preparations to decrease the impacts of Group 1 (nulliparous patients with single pregnancy and head presentation), Group 2 (nulliparous patients with single pregnancy and 
head presentation, term, before labor) and Group 5 (multiparous patients with at least one uterine scar and head presentation, $\geq 37$ weeks of gestation). These are the practices for not to increase the rates of primary cesarean section such as being insistent for labor induction before deciding cesarean section, not considering tubal ligation as a facilitator indication for cesarean section indication and performing external cephalic version in appropriate patients with breech presentation. In addition, providing appropriate conditions for vaginal delivery after cesarean section is another possible practice to decrease current rate of cesarean section. However, considering labor only as a medical practice and procedure is not a proper and sufficient approach; it will be the best to evaluate it within social, legal and systemic aspects in order to bring the approach and the rates of cesarean section into the optimal limits. ${ }^{[1]}$

Conflicts of Interest: No conflicts declared.

\section{References}

1. Cunningham FG, Leveno KJ, Bloom SL, Spong CY, Dashe JS, Hoffman BL, et al. Cesarean section and postpartum hysterectomy In: Williams obstetrics. 24th ed. NewYork: McGraw- Hill Education; 2014. p. 587-8.

2. T.C. Sağglı Bakanlığı Ana Çocuk Sağlı̆̆ı ve Aile Planlaması Genel Müdürlüğü. Doğum ve sezaryen eylemi yönetim rehberi. Ankara: Damla Matbaacılık; 2010.

3. WHO. Appropriate technology for birth. Lancet 1985;2: 436-7.

4. Souza JP, Betran AP, Dumont A, De Mucio B, Gibbs Pickens CM, Deneux-Tharaux C, et al. A global reference for caesarean section rates (C-Model): a multi country crosssectional study. BJOG 2016;123:427-36.

5. Hacettepe Üniversitesi Nüfus Etütleri Enstitüsü. 2013 Türkiye Nüfus ve Sağlık Araştırması. Ankara: Hacettepe Üniversitesi Nüfus Etütleri Enstitüsü, T.C. Kalkınma Bakanlığı ve TÜBİTAK; 2014. s. 150.

6. T.C. Sağglık Bakanlı̆̆ı. Sağlık İstatistikleri Y1llı̆g 2015. Ankara: T.C. Sağlık Bakanlığı; 2015. Retrieved from: www. sagem.gov.tr/dosyalar/SIY_2015.pdf

7. Mathai M, Hofmeyr GJ, Mathai NE. Abdominal surgical incisions for caesarean section. Cochrane Database Syst Rev 2013;(5):CD004453.

8. Begum T, Rahman A, Nababan H, Hoque DME, Khan AF, Ali T, et al. Indications and determinants of caesarean section delivery: evidence from a population-based study in Matlab, Bangladesh. PLoS One 2017;12:e0188074.

9. Betrán AP, Temmerman M, Kingdon C, Mohiddin A, Opiyo $\mathrm{N}$, Torloni $\mathrm{MR}$, et al. Interventions to reduce unnecessary caesarean sections in healthy women and babies. Lancet 2018; 392:1358-68.
10. T.C. Sağlık Bakanlığı. Doğum ve sezaryen eylemi yönetim rehberi. Ankara: T.C. Sağlık Bakanlı̆̆1; 2010. Retrieved from: http://sbu.saglik.gov.tr/Ekutuphane/kitaplar/a\%C3\%A7sap27. pdf

11. Robson MS. Can we reduce the caesarean section rate? Best Pract Res Clin Obstet Gynaecol 2001;15:179-94.

12. WHO. Robson classification: implementation manual. Geneva: WHO; 2017. Retrived from: https://www.who.int/ reproductivehealth/publications/maternal_perinatal_health/ robson-classification/en/

13. FIGO Working Group on Challenges in Care of Mothers and Infants During Labour and Delivery. Best practice advice on the 10-Group Classification System for cesarean deliveries. Int J Gynaecol Obstet 2016;135:232-3.

14. Cagan M, Tanacan A, Aydin Hakli D, Beksac MS. Changing rates of the modes of delivery over the decades (1976, 1986, 1996, 2006, and 2016) based on the Robson-10 group classification system in a single tertiary health care center. J Matern Fetal Neonatal Med 2019:1-8.

15. Ono T, Matsuda Y, Sasaki K, Satoh S, Tsuji S, Kimura F, et al. Comparative analysis of cesarean section rates using Robson Ten-Group Classification System and Lorenz curve in the main institutions in Japan. J Obstet Gynaecol Res 2016; 42:1279-85.

16. Gerli S, Favilli A, Franchini D, De Giorgi M, Casucci P, Parazzini F. Is the Robson's classification system burdened by obstetric pathologies, maternal characteristics and assistential levels in comparing hospitals cesarean rates? A regional analysis of class 1 and 3. J Matern Fetal Neonatal Med 2018;31: $173-7$.

17. Guida JP, Pacagnella RC, Costa ML, Ferreira EC, Cecatti JG. Evaluating vaginal-delivery rates after previous cesarean delivery using the Robson 10-group classification system at a tertiary center in Brazil. Int J Gynaecol Obstet 2017;136:354-5.

18. Triunfo S, Ferrazzani S, Lanzone A, Scambia G. Identification of obstetric targets for reducing cesarean section rate using the Robson Ten Group Classification in a tertiary level hospital. Eur J Obstet Gynecol Reprod Biol 2015;189:91-5.

19. Tan JK, Tan EL, Kanagalingan D, Tan LK. Rational dissection of a high institutional cesarean section rate: an analysis using the Robson Ten Group Classification System. J Obstet Gynaecol Res 2015;41:534-9.

20. Ferreira EC, Pacagnella RC, Costa ML, Cecatti JG. The Robson ten-group classification system for appraising deliveries at a tertiary referral hospital in Brazil. Int J Gynaecol Obstet 2015;129:236-9.

21. Abdel-Aleem H, Darwish A, Abdelaleem AA, Mansur M. Usefulness of the WHO C-Model to optimize the cesarean delivery rate in a tertiary hospital setting. Int J Gynaecol Obstet 2017;137:40-4.

22. Hanson C, Betrán AP, Opondo C, Mkumbo E, Manzi F, Mbaruku G, et al. Trends in caesarean section rates between 
2007 and 2013 in obstetric risk groups inspired by the Robson classification: results from population-based surveys in a lowresource setting. BJOG 2019;126:690-700.

23. Erkal Aksoy Y, Sert E, Er, M, Öztrük Can H. Robson On Gruplu Sinıflandırma Sistemine göre sezaryen doğumların incelenmesi. Uluslararası Hakemli Hemşirelik Araştırmaları Dergisi (UHD) 2015;(3):17-25

24. Süzer Özkan F, Demirci N. Factors affecting delivery preference of primigravida women: a qualitative study. Turkiye Klinikleri Journal of Nursing Science 2018;10:103-14.

25. Sarıkaya E(Ed.). Doğum şekli tercihinin multidisipliner irdelenmesi çalıştay kitapçığı. Ankara: TACESE; 2017. Retrieved from: https://www.tuseb.gov.tr/uploads/tacese_calistay_kitapcigi.pdf

26. Robson M, Hartigan L, Murphy M. Methods of achieving and maintaining an appropriate caesarean section rate. Best Pract Res Clin Obstet Gynaecol 2013;27:297-308.
27. ACOG Practice Bulletin No. 205: Vaginal birth after cesarean delivery. Obstet Gynecol 2019;133:e110-e127.

28. Hofmeyr GJ, Hannah M, Lawrie TA. Planned caesarean section for term breech delivery. Cochrane Database Syst Rev 2015;(7):CD000166.

29. Tsakiridis I, Mamopoulos A, Athanasiadis A, Dagklis T. Management of breech presentation: a comparison of four national evidence-based guidelines. Am J Perinatol 2019 Jun 5. doi:10.1055/s-0039-1692391

30. Singh R, Nath Trivedi A. Is the caesarean section rate a performance indicator of an obstetric unit? J Matern Fetal Neonatal Med 2011;24:204-7.

31. Türk Perinatoloji Derneği. Doğum eylem planı 2019-2023. İstanbul: Türk Perinatoloji Derneği; 2019. Retrieved from: http://cdn.perinatal.org.tr/files/TPD/sezeryan_TPD\%20 gorusu_2019.pdf

Bu makalenin kullanım izni Creative Commons Attribution-NoCommercial-NoDerivs 3.0 Unported (CC BY-NC-ND3.0) lisansı aracılığıly bedelsiz sunulmaktadır. / This work is licensed under the Creative Commons Attribution-NonCommercial-NoDerivs 3.0 Unported (CC BY-NC-ND3.0) License. To view a copy of this license, visit http://creativecommons.org/licenses/by-nc-nd/3.0/ or send a letter to Creative Commons, PO Box 1866 , Mountain View, CA 94042, USA. 